\title{
Muon cooling and acceleration
}

\author{
Masashi Otani (10)
}

\begin{abstract}
Muons, which were discovered in the 1930s and first generated using an accelerator half a century after their discovery, are now widely used in several scientific fields such as particle physics and material science. Recent advancements in cooling techniques have reduced the phase-space volume of muon beams and driven the effort to realize muon acceleration, which has now been demonstrated for the first time. This paper reviews the current state of muon cooling and acceleration technologies.
\end{abstract}

Keywords: Muon, Cooling, Accelerator, Linac

\section{Introduction}

A muon is an elementary particle similar to an electron, with an electric charge of $-e$ and a spin of $\frac{1}{2}$; however, the former has a mass 200 times heavier. Muons were discovered during the study of cosmic rays in the first half of the 20th century [1]. After the successful generation of muons using a particle accelerator half a century after their discovery [2], they have become widely used in various scientific fields.

Because muons have a heavier mass than electrons, they can easily couple with unknown particles through quantum effects. Elementary particle experiments such as the search for muon-to-electron conversion [3, 4] and precise measurement of the anomalous magnetic moment $[5,6]$ are currently in progress. Furthermore, muonium atoms (Mu: $\mu^{+} e^{-}$-bound state), because of their uniqueness as a pure leptonic system, can be one of the best probes for testing the precise bound-state QED and fundamental constants, for example, muon mass, muon magnetic moment, and fine structure constant [7-9]. Muon spin rotation/relaxation/resonance ( $\mu \mathrm{SR})$ is a collection of methods that use the muon's spin to examine structural and dynamical processes in bulk materials at the atomic scale. The spin motion of the muon inside the material provides information on its local environment in a similar way to other magnetic resonance techniques such as

Correspondence: masashio@post.kek.jp

Accelerator Laboratory, High Energy Accelerator Research Organization (KEK), Oho 1-1, Tsukuba 319-1106, Ibaraki, Japan nuclear magnetic resonance. In contrast to other methods, the signal of $\mu \mathrm{SR}$ can be easily detected even in polycrystalline samples; therefore, it is used to study the magnetism of high-temperature superconductors. Characteristic X-rays from the muonic atom (an atom wherein a negatively charged muon is captured by a nucleus) offer a unique method for non-destructive elemental analysis. This is because muonic $\mathrm{X}$-rays possess very high energy; thus, elemental analysis deep inside of a bulk material is possible. Thanks to a strong penetrating power of muons, cosmic-ray muons have been widely used for transmission imaging of large to medium scale structures such as pyramids [10], nuclear reactors [11], and containers.

All these applications could benefit from better quality muon beam. Because a muon beam is generated as a tertiary beam and initially has a large phase-space volume, cooling techniques have been developed to reduce the phase space volume, thus increasing the quality of the muon beam. These recent advancements have also provided a means to achieve muon acceleration with uses across numerous scientific applications.

Particle physicists are planning the construction of a muon collider, where muons are accelerated to a high energy before collision $[12,13]$. Another promising application of muon acceleration is the construction of a transmission muon microscope (https://slowmuon.kek. jp/index_e.html) for material and life sciences applications. If muons can be cooled to thermal temperature and subsequently re-accelerated, transmission muon microscopes can be realized. In addition, muon acceleration can 
be used in imaging. Although imaging with cosmic-ray muons is a unique way to see through large or shielded structures, the resolution and required time for imaging with muons are inadequate because of the muon flux and energy spread. Scattering tomography [14], which measures the muon scattering angle, depends not only on the amount of material but also on the muon energy. Accelerated muon beams are monochromatic and high-energy, enabling higher resolution imaging in less time.

Section 3 provides a detailed review of muon cooling techniques and Section 4 examines muon acceleration.

\section{Muon generation}

The muon is produced by decay of charged pions generated by the nuclear interaction in the production target with a proton beam. Recent remarkable progress on the accelerator technologies enable several MW proton beam for the usage of the muon production. Among several schemes or choices for the accelerators [15], the Japan Proton Accelerator Research Complex (J-PARC) and the Material and Lift Science Facility (MLF) in J-PARC is introduced because the J-PARC MLF is a lead facility on muon research.

The J-PARC accelerator is comprised of the $400-\mathrm{MeV}$ $\mathrm{H}^{-}$linear accelerator (linac), 3- $\mathrm{GeV}$ rapid-cycling synchrotron (RCS), and $30-\mathrm{GeV}$ main ring. The J-PARC linac provides beams with a design peak current of $50 \mathrm{~mA}$ with a macropulse duration of 500 us. The radio-frequency deflector combined with the scraper system is installed after the 3-MeV radio-frequency quadrupole linac (RFQ), in order to produce a so-called intermediate pulse configuration for the RCS. The $\mathrm{H}^{-}$beam is injected to the RCS via a charge-exchange injection through a thin carbon foil. After multuturn injection and acceleration in the RCS, most of the beam pulses are delivered to the MLF. The RCS has successfully demonstrated 1-MW beam operation for $36 \mathrm{~h}$ in summer 2020. The beam power at MLF is scheduled to be gradually increased to the primary goal of $1 \mathrm{MW}$ for use by experiments.

The MLF consists of muon and spallation neutron facilities, MUSE [16] and JSNS. The muon production target, a $2-\mathrm{cm}$ thick rotating graphite, installed $30 \mathrm{~m}$ upstream of the neutron target. There are four muon beamlines in MUSE. Two of the beamlines ( $\mathrm{H}$-line and $\mathrm{D}$-line) are extracted at the angle of 60 degrees to the proton beamline, and the others (S-line and U-line) are at 135 degrees. The D-line [17] collects muons or pions with three quadrupole magnets with a solid angle of $65 \mathrm{msr}$. Decay positive and negative muons up to $120 \mathrm{MeV} / \mathrm{c}$ can be extracted by using a superconducing solenoid magnet and a typical intensity is an order of $10^{6}$ muons per seconds at $60 \mathrm{MeV} / \mathrm{c}$. The D-line has been operated for user program from 2008. The U-line [18] uses the capture solenoid with a large solid angle acceptance of $400 \mathrm{msr}$ and transports muons using solenoids with two curved sections of 45 degree each of opposite direction and a 6-m long straight section. The U-line is was installed in 2012 and being operated to generate the ultra-slow muons with the muonium laser ionization described in Chap. 3. The S-line [19] collects and transports muons with quadrupole magnets. It will eventually comprise four experimental areas that will share the beam via electric kickers. The $\mathrm{H}$-line [20] aims to realize a high-intensity muon beam using a large aperture capture solenoid with a solid angle of $136 \mathrm{msr}$. The two short $1.5 \mathrm{~m}$ solenoid magnets with fields that are mutually in opposite directions are adopted for the beam transport. The expected beam intensity at the experimental area is approximately $10^{8}$ muons per seconds and the beam commissioning will be conducted in 2022.

\section{Muon cooling}

The cooling techniques developed at particle accelerators, synchrotron radiation cooling, laser cooling [21, 22], stochastic cooling [23] and electron cooling [24] cannot be used for muons because the required time for cooling is significantly longer than the muon lifetime of $2 \mu \mathrm{s}$. The most practical approach for muon cooling is to pass muons through a particular material.

Ionization cooling [25] is a method of beam cooling wherein charged beam particles lose kinetic energy as they pass through a certain material. Acceleration of the muons using radio-frequency cavities after deceleration in the material restores momentum only along the beam direction. This process is repeated several times to reduce the transverse momentum. The emittance change during this process can be expressed as

$$
\frac{d \varepsilon}{d z} \approx-\frac{\varepsilon}{\beta^{2} E} \frac{d E}{d z}+\frac{\beta_{\perp}(13.6[\mathrm{MeV} / \mathrm{c}])^{2}}{2 \beta^{2} E m X_{0}}
$$

where $\beta, E, m$ are the muon velocity, energy, and mass of muon, respectively. $\varepsilon$ is the transverse emittance, $\beta_{\perp}$ is the transverse beta function, and $X_{0}$ is the radiation length of the material. In order to reduce the heating effect expressed in the second term, low- $Z$ material (small $X_{0}$ ) is desired as the deceleration material. The international muon ionization cooling experiment (MICE http://mice. iit.edu) recently confirmed the principle of this method experimentally. In the experiment, the phase space distributions in the transverse directions before and after both liquid hydrogen and lithium hydride absorbers are measured using scintillating fiber layers. They successfully observed emittance reduction as expected [26]. This method of cooling is generally useful for muon energies larger than few hundred $\mathrm{keV}$, where the energy loss per unit length is given by the Bethe formula.

For particles moving more slowly than $\beta=v / c \sim 0.01$, the stopping power becomes approximately proportional 
to $\beta[27,28]$. In this energy region, the moderator and acceleration along the muon path increase the spectral density around a certain equilibrium energy, like the ionizaion cooling. The experimental proof for this frictional cooling was found in [29]. In the experiment, muons pass a series of stainless ring where the acceleration voltage are applied. Some of the rings install a thin graphite foil, through which the cooling was achieved. A total increase of the phase-space density for muons with energies from 0.5 to $1.5 \mathrm{keV}$ is observed to be a factor of $12_{+9}^{-5}$. Additional methods for more efficient cooling were proposed by combining ionization cooling with a large admittance realized by an inverse cyclotron system [30].

For muon energies of less than a few $\mathrm{keV}$, or velocities smaller than valence electron velocities, there are drastic differences between the behavior of negative and positive muons. One of these differences is the Barkas correction [31], which is necessary for describing the stopping power for negative particles in matter. Another important difference is that a positive muon can capture an electron from a material to form a $\mathrm{Mu}$ or two electrons to form a negative muonium ion $\left(\mu^{+} e^{-} e^{-}, \mathrm{Mu}^{-}\right)$.

The cold moderator method was successfully used at the Paul Scherrer Institute (PSI) to generate slow $(1 \sim 10 \mathrm{eV})$ $\mu^{+}$beams [32, 33]. Because several cryocrystals have a large energy gap ( $\sim 10 \mathrm{eV}$ for $\mathrm{Ar}$ and $\mathrm{N}_{2}$, for example), the energy loss process is terminated at that energy and the slow $\mu^{+}$will be emitted from the surface of the cryocrystal injection target. In this method, the produced low $\mu^{+}$ maintains almost $100 \%$ polarization; however, the conversion efficiency from the injected few $\mathrm{MeV}$ of $\mu^{+}$to slow $\mu^{+}$is $\sim 10^{-5}$.

At PSI, the new method based on an energy loss during the cryogenic gas has been developed. The decelerated muons are transported with electric and magnetic fields in combination with gas density gradients, and $\sim 10 \mathrm{eV}$ muons are obtained. Recently, the muCool collaboration succeeded in demonstrating transverse compression and improving the phase-space density by a factor of $10^{10}$ with $10^{-3}$ efficiency [34].

The muonium laser ionization technique can generate positive muons with thermal energy. In this method, conventional beam muons are injected into the $\mathrm{Mu}$ production target and then form thermal $\mathrm{Mu}$. The paired electron in $\mathrm{Mu}$ is knocked out by a laser, and a thermal muon is generated (Fig. 1). After first observation of the muonium in vacuum with a hot tungsten foil [35], ionization of the muonium via the excitation of the two-photon transition $1 \mathrm{~s} \rightarrow 2 \mathrm{~s}$ [36] and single photon transition $1 \mathrm{~s} \rightarrow 2 \mathrm{p}$ [37] were achieved. In the RIKEN-RAL muon facility located at Rutherford Appleton Laboratory, the thermal muon intensity was achieved to be $15 \mu^{+}$per second and the efficiency of the surface muon cooling to thermal energy is order of $10^{-5}$ [38]. Because the cooling efficiency above is limited mainly by the overlap between the muonium spread and laser region, the efficiency is potentially possible to be the probability of the muonium production, the order of $10^{-1} \sim 10^{-2}$. Recent progress using a silica aerogel target as the muonium production target showed an order-of-magnitude improvement of the muonium emission from the target with the laser ablation technique $[39,40]$.

$\mathrm{Mu}^{-}$was observed in vacuum for the first time in the late 1980s [41-43]. In this method, the conversion efficiency from $\mu^{+}$to $\mathrm{Mu}^{-}$with the energy smaller than $1 \mathrm{keV}$ is limited to the order of $10^{-5}$. It has been theorized, since the first $\mathrm{Mu}^{-}$was generated, that the conversion efficiency would be enhanced with materials with a

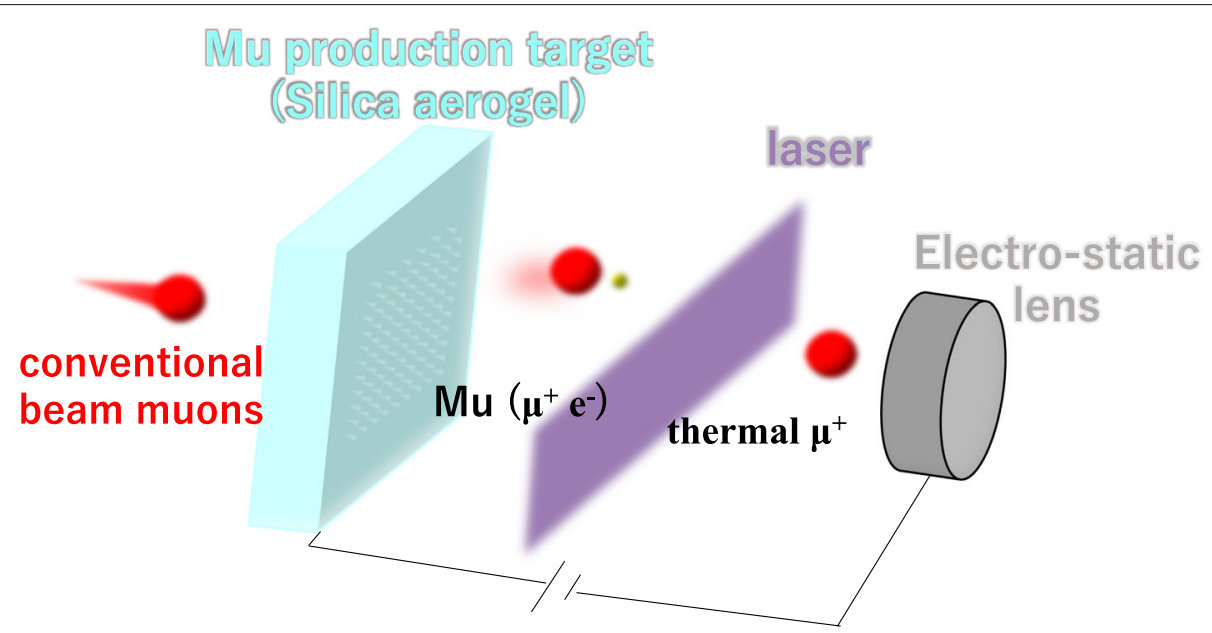

Fig. 1 Schematic of the muonium laser ionization technique. The conventional beam muons are injected into a Mu production target. Some of the Mu will escape to vacuum with thermal energy. The laser strips the electron from Mu, and the muon with thermal energy is generated 
low-work function $[41,44]$. The demand for higher efficiency conversion has spurred the development of a radiofrequency linear accelerator $\mathrm{Mu}^{-}$generation method. Several experiments have been conducted in MLF at JPARC $[45,46]$. In the experiments, the $\mathrm{Mu}^{-}$generation efficiency was measured using several targets: C12A7 electrode $[47,48], \mathrm{Al}$ foil, and SUS foil. The $\mathrm{Mu}^{-}$intensity is consistent within the statistical uncertainty of $\sim 10 \%$ among the three targets. This systematic measurement is essential input to understand the $\mathrm{Mu}^{-}$production process.

As described above, $\mu^{-}$behaves differently to $\mu^{+}$at energies below a few hundred keV. Because the muonic atom is formed and no re-emission is expected, it is difficult to produce slow $\mu^{-}$. To overcome this difficulty, it was proposed to utilize the muon catalyzed fusion $(\mu \mathrm{CF})$ process described below $[49,50]$. As a first step of $\mu \mathrm{CF}, \mu^{-}$is injected to a deuterium-tritium (D-T) mixture and forms either $\mathrm{d} \mu$ or $\mathrm{t} \mu$ atom. The generated $\mathrm{t} \mu$ acts with deuterium atom in the molecules to form $\mathrm{dt} \mu$ and $\mu^{-}$with energy of around $10 \mathrm{keV}$ will be released after the fusion reaction of $\mathrm{d}+\mathrm{t}$. Currently, a proof-of-principle experiment is being planned for J-PARC.

\section{Muon acceleration}

As described in Section 3, ionization cooling requires rapid acceleration with a radio-frequency cavity, in addition to deceleration in the absorbers. In this method, it is necessary to accommodate the cavity in the strong solenoid field in order to focus the muon beam with large emittance and realize a small transverse divergence for efficient cooling. In axial magnetic fields, electronloading phenomena in the acceleration cavity, such as multipathing and electrical breakdown, are enhanced. To mitigate these phenomena, testing on the operation of the cavity in a high magnetic field has been conducted at the Fermilab MuCool Test Area (MTA). Operation of a cavity with more than $50 \mathrm{MV} / \mathrm{m}$ in a 3-T magnetic field was achieved using Beryllium end plates that have higher resistance to the expected electrical breakdown [51, 52]. Another attempt using a high pressure gas-filled radio-frequency cavity, in which the induced electrons are terminated by the collision with gas molecules, also succeeded in the operation with similar parameters [53].

The acceleration of muons using a radio-frequency accelerator was demonstrated for the first time [54] in the J-PARC MLF. In this experiment, the $\mathrm{Mu}^{-}$is produced by injecting surface muons into a thin metal foil. After generation and extraction with an electro-static lens, the $\mathrm{Mu}^{-}$ is injected into an RFQ [55] and accelerated to $89 \mathrm{keV}$. The $\mathrm{Mu}^{-}$is then transported to the microchannel plate detector through the diagnostic beamline consisting of two quadrupole magnets and a bending magnet. In addition to the charge and momentum selection by the bending magnet, the time-of-flight (ToF) from the $\mathrm{Mu}^{-}$production target to the detector is measured as an additional figure of merit for the accelerated $\mathrm{Mu}^{-}$. Figure 2 shows

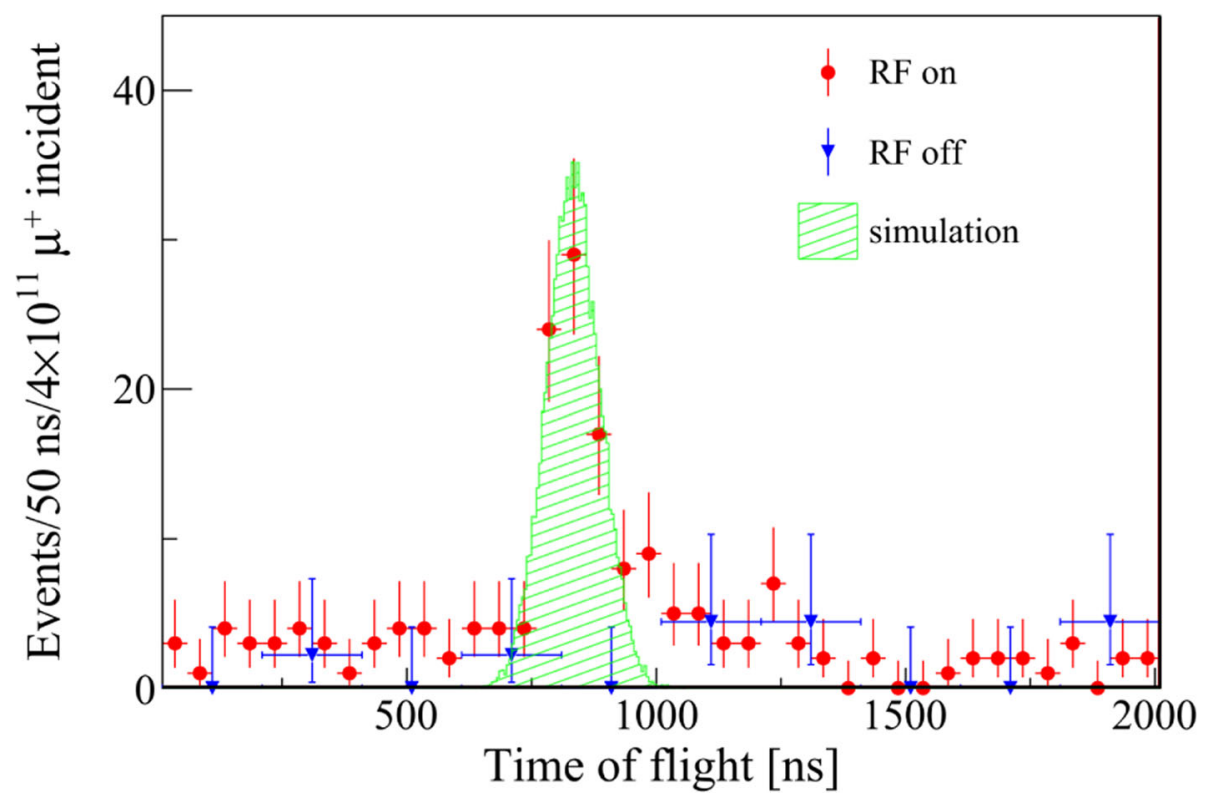

Fig. 2 The ToF spectra of ovserbed $\mathrm{Mu}^{-}$events with RFQ on and off. Because the $\mathrm{Mu}^{-}$is produced by injecting $\mu^{+}$into a thin metal foil, the number of the $\mathrm{Mu}^{-}$events is normalized with the number of injected $\mu^{+}$events. The clear peak of the RFQ on the spectrum at $830 \mathrm{~ns}$ corresponds to the accelerated $\mathrm{Mu}^{-}$particles. The error bars are statistical. A simulated ToF spectrum of the accelerated $\mathrm{Mu}^{-}$'s is also plotted. This figure is cited from [54] 


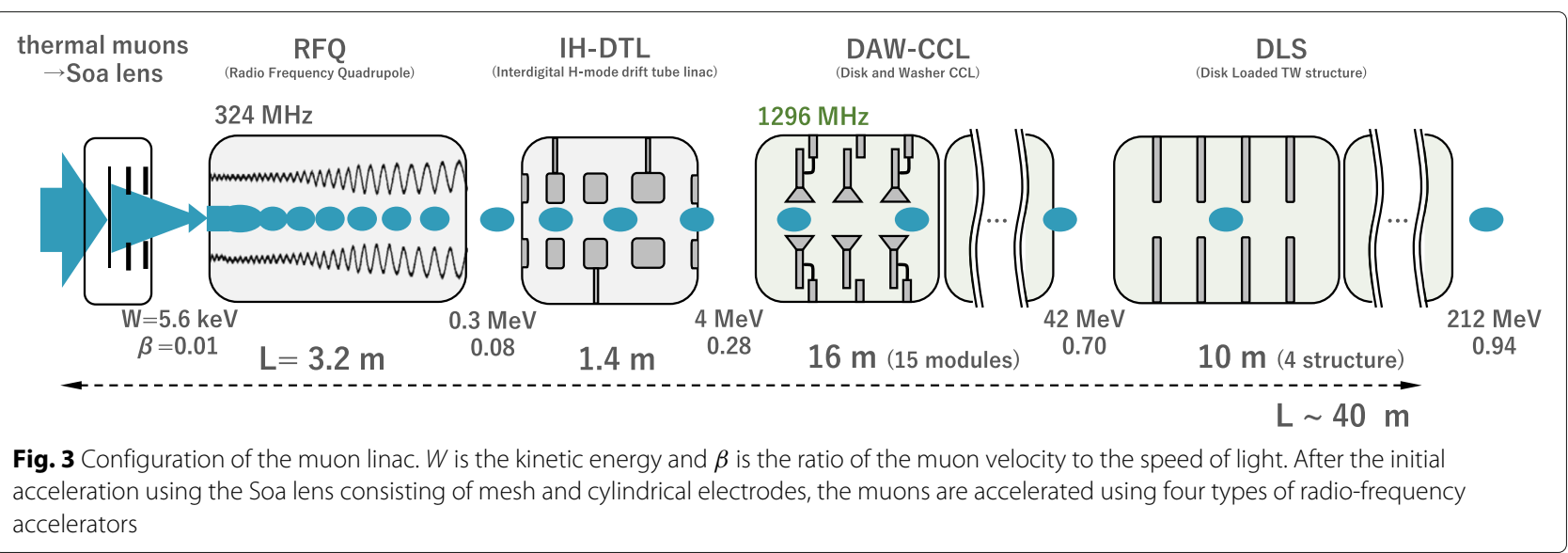

the ToF spectrum with and without RFQ operation after the signal pulse-height cut is applied [56]. The hatched histogram shown in Fig. 2 represents the simulated ToF spectrum of the accelerated $\mathrm{Mu}^{-}$which is consistent with the measured ToF values. It can be concluded from these results that the observed peak is due to the acceleration of $\mathrm{Mu}^{-}$by the RFQ to $89 \mathrm{keV}$.

A linac, based on the radio-frequency acceleration technology described above, is being developed at J-PARC to accelerate muons to nearly the speed of light in order to the measure the muon anomalous magnetic moment and electric dipole moment [6]. Figure 3 shows schematic of the muon linac. An RFQ bunches and accelerates the muons to $0.3 \mathrm{MeV}$ [57] after the initial acceleration using an electrostatic lens called a Soa lens [58]. After the $\mathrm{RFQ}$, an interdigital H-mode drift tube linac (IH-DTL) is employed while the particle velocity $\beta=0.08$ to 0.28 $(4 \mathrm{MeV})$ [59]. The IH-DTL was first proposed in Japan in 1949 [60]. Compared to the conventional Arvaretz DTL, a higher acceleration efficiency is achieved, particularly within the range of $\beta=0.1-0.2$ [61]. After the muon is accelerated to $\beta=0.28$, a disk-and-washer (DAW)-type coupled cavity linac (CCL) with an operational frequency of $1296 \mathrm{MHz}$ [62] is employed. A DAW-CCL was first proposed in the early 1970s [63, 64], and full scale cavity was demonstrated in late 1970s [65]; the first DAW-CCL was operated using protons and $\mathrm{H}^{-}$linac at the Moscow meson factory [66]. DAW-CCLs have advantages over other CCLs, such as a higher shunt impedance and higher coupling between the accelerating and coupling cells. A disk-loaded structure (DLS) traveling-wave linac is used when $\beta$ is greater than $0.7(42 \mathrm{MeV})$ [67]. DLS is classified as a traveling wave accelerator for electrons. The acceleration of electrons using a traveling wave accelerator was first demonstrated in the late 1940s [68], and recent electron accelerators using room-temperature cavities have been based on the results of the Mark III linac [69] and SLAC. Unlike linear accelerators for electrons, which quickly reach the speed of light, muon linacs slowly approach the speed of light and require a gradual change in the length of the cell. Prototypes of the IH-DTL and DAW-CCL were fabricated to confirm the design and evaluate the performance [70]. The actual IH-DTL and first module of the DAW-CCL are currently being fabricated.

To realize a mobile muon accelerator to inspect infrastructure such as roads, the accelerator requires compactness. One of the barriers to building such a device is the low velocity section of the muon accelerator; it requires approximately $10 \mathrm{~m}$ to accelerate muons to a few tens of $\mathrm{MeV}$ in the design described above. The automatic cyclotron resonance acceleration, which was utilized in electron acceleration [71, 72], has been discussed for proton acceleration [73] and muons. Because the muon mass is approximately one tenth that of the proton, the application of this acceleration scheme to muons decreases the strength of the required magnetic field. Figure 4 shows the simulation result for muon acceleration with the automatic cyclotron resonance scheme. A uniform magnetic field of $6.7 \mathrm{~T}$ is applied to the resonant cavity operating in the $\mathrm{TE}_{111}$ mode with a frequency of $850 \mathrm{MHz}$. This magnetic field can accelerate muons from $10 \mathrm{keV}$ to $20 \mathrm{MeV}$ with a length of $29 \mathrm{~cm}$, which is much shorter than the current designs. To achieve greater acceleration, more studies are needed, such as operating a high-power radio-frequency cavity in an axial magnetic field.

\section{Summary}

A wide variety of scientific fields have been explored using muon beams since their discovery. Recent progress in the cooling techniques of muon beams has driven the advancement of muon acceleration and led to the first successful demonstration of muon acceleration. Accelerated muon beams, utilizing advanced cooling techniques, have begun a new era of accelerator science. 


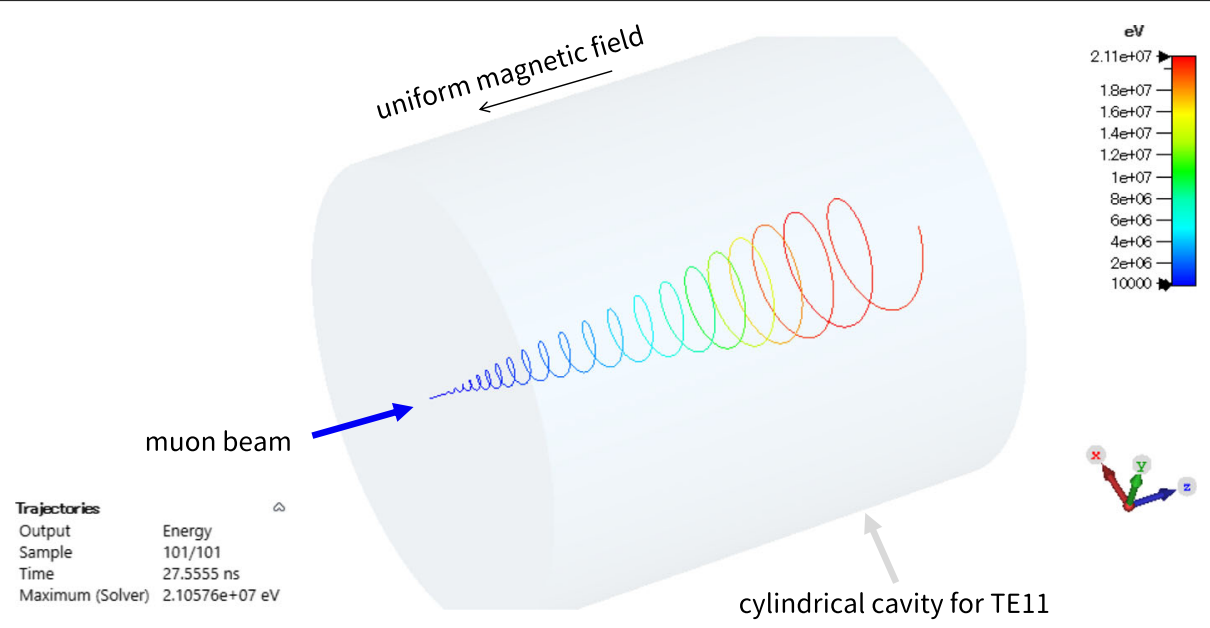

Fig. 4 Example of beam tracking simulation. The muon beam trajectory is shown with its energy

\section{Acknowledgments}

The author acknowledges the AAPPS-APCTP Chen-Ning Yang Award committee members for giving the valuable opportunity to write this article.

\section{Author's contributions}

The author wrote this review article by compiling information from the published papers from each experiment. The author read and approved the final manuscript.

\section{Author's information}

Masashi Otani is an assistant professor at Accelerator Laboratory, KEK. He has been involved in the muon $g-2$ /EDM experiment at J-PARC since 2013, leading the muon linac development for the experiment. He was awarded the AAPPS-APCTP Chen-Ning Yang Award (2021) for his development of the muon linac in realizing the muon acceleration for the first time in the world. His research field is accelerator and particle physics.

\section{Funding}

This work is supported by JSPS KAKENHI (Grant Numbers 15H03666, 18H03707, 21K18630, 21H05088), JST FOREST Program (Grant Number JPMJFR212O) and the natural science grant of the Mitsubishi Foundation.

\section{Availability of data and materials}

Not applicable.

\section{Declarations}

Ethical approval and consent to participate

Not applicable.

\section{Consent for publication}

Not applicable.

\section{Competing interests}

The author declares no competing interests.

Received: 22 December 2021 Accepted: 4 February 2022

Published online: 01 March 2022

\section{References}

1. S. H. Neddermeyer, C. D. Anderson, Note on the Nature of Cosmic-Ray Particles. Phys. Rev. 51, 884 (1937)

2. E. G. Michaelis, Review of meson factories. IEEE Trans. Nucl. Sci. NS-23(3), 1385 (1975)

3. L. Bartoszek, et al., Mu2e Technical Design Report. Fermilab-TM-2594 (2015)

4. The COMET Collaboration, COMET Phase-I technical design report. Prog Theor. Exp. Phys. 2020, 033C01 (2020)
5. B. Abi, et al., [Muon g-2 Collaboration]. Phys. Rev. Lett. 141801, 126 (2021)

6. M. Abe, et al., A new approach for measuring the muon anomalous magnetic moment and electric dipole moment. Prog. Theor. Exp. Phys. $\mathbf{2 0 1 9}, 053 \mathrm{C02}$ (2019)

7. P. Crivelli, Hyperfine Interact. 239(1), 49 (2018)

8. S. Kanda, et al., New precise spectroscopy of the hyperfine structure in muonium with a high-intensity pulsed muon beam. Phys. Lett. B. 136154, 815 (2021)

9. C. Zhang, et al., Simulation Study of Laser lonization of Muonium by $15-2 S$ Excitation for the Muon g - 2/EDM Experiment at J-PARC. JPS Conf. Proc. 011125, 33 (2021)

10. K. Morishima, M. Kuno, M. Tayoubi, Discovery of a big void in Khufu's Pyramid by observation of cosmic-ray muons. Nature. 552, 386-390 (2017)

11. C. L. Morris, et al., Analysis of muon radiography of the Toshiba nuclear critical assembly reactor. Appl. Phys. Lett. 104, 024110 (2014)

12. M. Boscolo, J. P. Delahaye, M. Palmer, The future prospects of muon colliders and neutrino factories. Rev. Acc. Sci. Tech. 10(01), 189-214 (2019)

13. M. Boscolo, et al., Low emittance muon accelerator studies with production from positrons on target. Phys. Rev. AB. 21, 061005 (2018)

14. K. Ralf, Muography: overview and future directions. Phil. Trans. R. Soc. A. 377, 20180049 (2019)

15. H. Hotchi, High-power proton accelerators for pulsed spallation neutron sources. AAPPS Bull. 31, 23 (2021)

16. Y. Miyake, et al., J-PARC Muon Facility, MUSE. JPS Conf. Proc. 21, 011054 (2018)

17. P. Strasser, et al., J-PARC decay muon channel construction status. JPS Conf. Proc. 225, 012050 (2010)

18. Y. Ikedo, et al., U-Line at MLF/J-PARC for Ultra Slow Muon Microscopy. JPS Conf. Proc. 2, 010103 (2014)

19. P. Strasser, et al., Status of the New Surface Muon Beamline at J-PARC MUSE. JPS Conf. Proc. 21, 011061 (2018)

20. N. Kawamura, et al., New concept for a large-acceptance general-purpose muon beamline. Prog. Theor. Exp. Phys. 2018, 113G01 (2018)

21. T. Hänsh, A. Schawlow, Cooling of gases by laser radiation. Opt. Commun. 13, 68 (1975)

22. D. J. Wineland, H. Dehmelt, Proposed $10^{14} \mathrm{Dn}<n$ laser fluorescence spectroscopy on. $\mathrm{TI}^{+}$mono-ion oscillator III. Bull. Am. Phys. Soc. 20, 637 (1975)

23. S. van der Meer, Stochastic damping of betatron oscillations in the ISR. CERN-ISR-PO-72-31 (1972)

24. R. B. Palmer, Stochastic cooling. Brookhaven Nat. Lab.internal Report BNL, 18395 (1973)

25. M. Ado, V. I. Baldekov, Use of ionization friction in the storage of heavy particles. Sov. At. Energy. 31, 731 (1971)

26. MICE collaboration, Demonstration of cooling by the Muon Ionization Cooling Experiment. Nature. 578, 53-59 (2020)

27. J. Lindhard, On the properties ofa gas of charged particles. Kgl Mat.-Fys. Medd. 28, 8 (1954) 
28. J. Lindhard, et al., Range concepts and heavy ion ranges. Kgl. Danske Videnskab. Selskab, Mat.-Fys. Medd. 33, 14 (1963)

29. M. Mühlbauer, et al., Hyperfine Interact. 119, 305-310 (1999)

30. T. Hart, et al., in Proceedings of Cyclotrons2013. An Inverse Cyclotron for Muon Cooling (The Joint Accelerator Conferences Website (JACoW), Vancouver, 2013), pp. 97-101

31. R. Schmidt, et al., Measurement of the Barkas effect in hydrogen. Eur. Phys. J. D. 3, 119-122 (1998)

32. D. R. Harshman, Observation of Low-Energy $\mu+$ Emission from Solid Surfaces. Rev. Phys. Lett. 56, 2850 (1986)

33. E. Morenzoni, et al., Generation of very slow polarized positive muons. Phys. Rev. Lett. 72, 2793 (1995)

34. A. Antognini, et al., Demonstration of Muon-Beam Transverse Phase-Space Compression. Phys. Rev. Lett. 125, 164802 (2020)

35. A. P. Mills, et al., Generation of Thermal Muonium in Vacuum. Phys. Rev. Lett. 56, 1463 (1986)

36. S. Chu, et al., Laser Excitation of the Muonium 1S-2S Transition. Phys. Rev. Lett. 60, 101 (1988)

37. K. Nagamine, et al., Ultraslow Positive-Muon Generation by Laser Ionization of Thermal Muonium from Hot Tungsten at Primary Proton Beam. Phys. Rev. Lett. 74, 4811 (1995)

38. P. Bakule, et al., Pulsed source of ultra low energy positive muons for near-surface $\mu$ SR studies. Nucl. Instr. Meth. 266, 335 (2008)

39. G. A. Beer, et al., Enhancement of muonium emission rate from silica aerogel with a laser-ablated surface. Prog. Theor. Exp. Phys. 2014, $091 \mathrm{C} 01$ (2014)

40. J. Beare, K. Suzuki, et al., Study of muonium emission from laser-ablated silica aerogel. Prog. Theor. Exp. Phys. 2020, 123 C01 (2020)

41. Y. Kuang, et al., Formation of the negative muonium ion and charge-exchange processes for positive muons passing through thin metal foils. Phy. Rev. A39, 6109 (1989)

42. Y. Kuang, et al., First observation of the negative muonium ion produced by electron capture in a beam-foil experiment. Phy. Rev. A35, 3172 (1987)

43. D. R. Harshman, et al., Observation of Low-Energy ?+ Emission from Solid Surfaces. Phy. Rev. Lett. 56, 2850 (1986)

44. V. G. Dudnikov, et al., in Proceedings of 8 th International Particle Accelerator Conference. Cold Muonium Negative Ion Production, (Copenhagen, 2018), p. 2898

45. M. Otani, et al., Negative Muonium Ion Production With a C12A7 Electride Film. J. Phys. Conf. Ser. 1350, 012067 (2019)

46. R. Kitamura, et al., Development of negative muonium ion source for muon acceleration. Phys. Rev. AB. 24, 033403 (2021)

47. H. Hosono, et al., High-Density Electron Anions in a Nanoporous Single Crystal: [Ca24Al28064]4+(4e-). Science. 301, 626 (2003)

48. H. Hosono, et al., Transparent amorphous oxide semiconductors for organic electronics: Application to inverted OLEDs. Proc. Natl. Acad. Sci USA. 114(2), 233 (2017)

49. K. Nagamine, Ultra-slow Negative Muon Production via Muon Catalyzed Fusion. Proc. Jpn Acad. 65B, 225 (1989)

50. K. Nagamine, Introductory Muon Science. (Cambridge University Press, Cambridge, 2003)

51. M. Palmer, et al., Reviews of Accelerator Science and Technology. RAST. 10(01), 189-214 (2019)

52. D. Bowring, et al., Operation of normal-conducting rf cavities in multi-Tesla magnetic fields for muon ionization cooling: A feasibility demonstration. Phys. Rev. AB. 23, 072001 (2020)

53. M. Chung, et al., Pressurized $\mathrm{H} 2$ rf Cavities in lonizing Beams and Magnetic Fields. Phys. Rev. Lett. 111, 184802 (2013)

54. S. Bae, et al., First muon acceleration using a radio-frequency accelerator. Phys. Rev. AB. 21, 050101 (2018)

55. Y. Kondo, K. Hasegawa, A. Ueno, Fabrication and low-power measurements of the J-PARC 50-mA RFQ prototype. Proc. LINAC. 2006, 749 (2006)

56. M. Otani, et al., Compact buncher cavity for muons accelerated by a radio-frequency quadrupole. Nucl. Inst. Meth. Phys. Res. Sec. 946, 162693 (2019)

57. Y. Kondo, et al., Simulation study of muon acceleration using RFQ for a new muon g-2 experiment at J-PARC. Proc. IPAC. 2015, 3801 (2015)

58. K. F. Canter, P. H. Lippel, W. S. Crane, A. P. Mills Jr, in Positron studies of solids, surfaces and atoms. Positron studies of solids, surfaces and atoms (World Scientific, Singapore, 1986), p. 199
59. M. Otani, et al., Interdigital H-mode drift-tube linac design with alternative phase focusing for muon linac. Phys. Rev. AB. 19, 040101 (2016)

60. H. Morinaga, Phys. Soc. Meeting, Osaka (1949)

61. U. Ratzinger, H-type linac structures. CERN Yellow Report. 2005-003, 351 (2005)

62. M. Otani, et al., Disk and Washer Coupled Cavity Linac Design and Cold-Model for Muon Linac. J. Phys. Conf. Ser. 1350, 012097 (2019)

63. V. G. Andrev, et al., Study of High-Energy Proton Linac Structures. Proc. Linear Acel. Conf. 1972, 114 (1972)

64. B. P. Murin, et al., High-Current Ion Linear Accelerator for Medium-Energy Physics (Meson Factory). Proc. Proton Linac Conf. 1972(LA-5115), 387 (1972)

65. V. G. Andreev, et al., Investigation of the Accelerating Structure for the Second Part of the Meson Factory Linac. Proc. Linac Conf. 1976(AECL-5677), 269 (1976)

66. S. K. Esin, et al., The Disk and Washer Structure for Moscow Meson Factory Linac. Proc. Linear Accel. Conf. 1988, 657 (1988)

67. Y. Kondo, et al., Beam dynamics design of the muon linac high-beta section. J. Phys. Conf. Ser. 012054, 875 (2017)

68. E. L. Ginzton, W. W. Hansen, W. R. Kennedy, A Linear Electron Accelerator. Rev. Sci. Instrum. 19, 89 (1948)

69. M. Chodorow, et al., Stanford High?Energy Linear Electron Accelerator (Mark III). Rev. Sci. Instrum. 26, 134 (1955)

70. Y. Nakazawa, et al., Development of Inter-Digital H-Mode Drift-Tube Linac Prototype With Alternative Phase Focusing for a Muon Linac in the J-PARC Muon G-2/EDM Experiment. J. Phys. Conf. Ser. 1350, 012054 (2019)

71. B. Hafizi, P. Sprangle, J. L. Hirshfield, Electron beam quality in a cyclotron autoresonance accelerator. Phys. Rev. E. 50, 3077 (1994)

72. M. A. LaPointe, R. B. Yoder, C. Wang, A. K. Ganguly, J. L. Hirshfield, Experimental Demonstration of High Efficiency Electron Cyclotron Autoresonance Acceleration. Phys. Rev. Lett. 76, 2718 (1996)

73. T. Hara, in Proceedings of the 17th Annual Meeting of Particle Accelerator Society of Japan. Conceptual design for proton accelerator with cyclotron auto-resonance (Particle Accelerator Society of Japan, 2020), pp. 43-45

\section{Publisher's Note}

Springer Nature remains neutral with regard to jurisdictional claims in published maps and institutional affiliations. 